\title{
Neuropsychological Impact of Bilateral Cerebral Abscesses in an Adolescent
}

\author{
Alice Ann Spurginin b, e, Paul C. Glasier ${ }^{\mathrm{a}, \mathrm{b}}$, Michael Morriss ${ }^{\mathrm{c}, \mathrm{d}}$, \\ Peter L. Stavinoha ${ }^{a, b}$
}

\begin{abstract}
Existing literature on neuropsychological outcomes of cerebral abscesses is limited to a single prior study in the adult population. To our knowledge, there is no existing study of neuropsychological outcomes of pediatric cerebral abscesses. Additionally, to our knowledge, no such studies of cerebral abscesses in either a pediatric or an adult population have been published in North America. Through presentation of comprehensive neuropsychological and imaging data, this case of pediatric cerebral abscesses demonstrates the anatomical and neuropsychological impact of right frontal and left thalamic Streptococcus intermedius infection in an adolescent with a history of complex congenital heart disease, a known risk factor for cerebral abscesses. Abscess localization is clearly connected to functional outcomes, specifically deficits in nonverbal reasoning, visual-motor integration, bilateral fine motor speed, motor-based processing speed, memory, and executive functioning. Recommendations are provided for post-acute neuropsychological evaluation of bilateral cerebral abscesses.
\end{abstract}

Keywords: Cerebral abscess; Streptococcus intermedius; Pediatrics; Neuropsychology

\section{Introduction}

Cerebral abscess, also described as focal intraparenchymal

Manuscript accepted for publication June 19, 2012

\footnotetext{
${ }^{a}$ Department of Psychiatry, University of Texas Southwestern Medical Center, Dallas 75235, U.S.A.

bepartment of Psychology, Children's Medical Center, Dallas 75235, U.S.A.

${ }^{\mathrm{c}}$ Department of Radiology, University of Texas Southwestern Medical Center, Dallas 75235, U.S.A.

${ }^{\mathrm{d} D e p a r t m e n t ~ o f ~ R a d i o l o g y, ~ C h i l d r e n ' s ~ M e d i c a l ~ C e n t e r, ~ D a l l a s ~ 75235, ~}$ U.S.A.

'Corresponding author: Alice Ann Spurgin, Children's Medical Center Neuropsychology, 6300 Harry Hines Blvd., Suite 900, Dallas, TX 75235, USA. Email: AliceAnn.Spurgin@utsouthwestern.edu
}

doi:10.4021/jnr104w infection, occurs in about four to ten out of every million people each year, most typically males between 4 and 7 years of age $[1,2]$. The higher frequency of cerebral abscess in young children is thought to be due to the higher frequency of contributing factors such as sinusitis, otitis, mastoiditis, and congenital heart disease (CHD), although in up to $30 \%$ of cases no source of infection can be determined [3]. Cerebral abscess is an acute, progressive, and nearly $100 \%$ fatal condition if untreated. Standard of care involves neurosurgical drainage of the pus filling the abscess and administration of antibiotics specific to the infectious agent. The earliest symptoms of cerebral abscess are typically nonlocalizing headache, nausea, and vomiting [1]. Although adults presenting with cerebral abscess are commonly afebrile, a retrospective study of 30 children found that all but a single neonate presented with fever [4]. Focal neurological signs depend on the localization of the abscess and may be absent in up to $50 \%$ of patients [1]. Progression can result in symptoms such as seizures and alterations in consciousness [1].

Diagnosis is established by CT or MRI, which has contributed to the reduction of mortality rates from $35-55 \%$ to

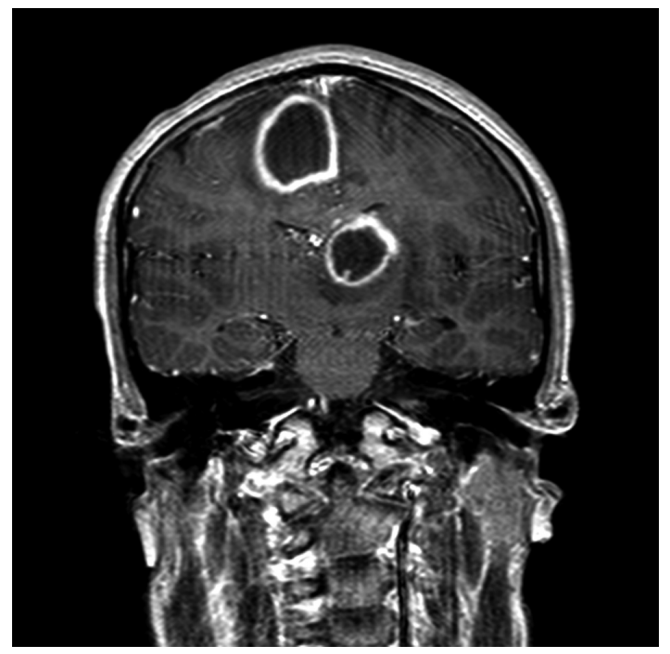

Figure 1. T1-weighted contrast-enhanced coronal image from the day of admission shows abscesses with typical thick-walled enhancement in left ventral thalamus and right superior frontal gyrus, just above the cingulate gyrus. 


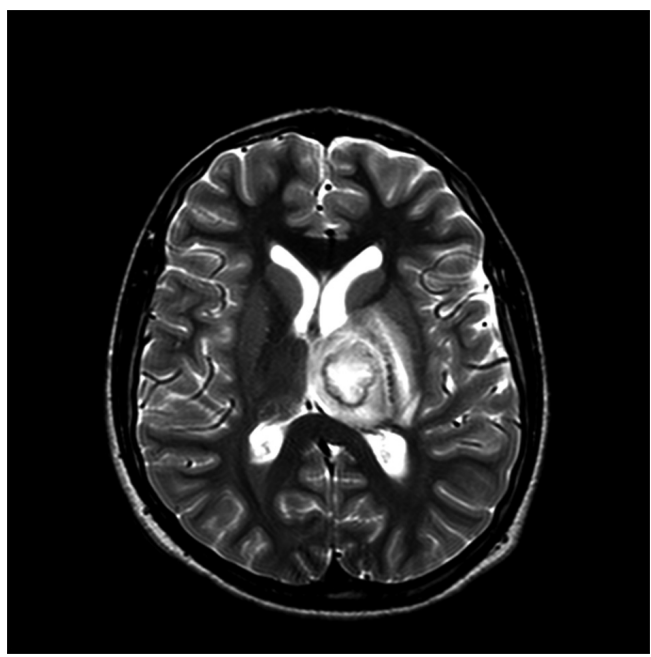

Figure 2. T2-weighted axial image from day of admission shows left thalamic abscess creating mass effect on the fornix and edema extending into the posterior limb of the internal capsule, causing mass effect on the left optic tract (not shown).

$0-30 \%$ [5]. Morbidity and mortality are significantly influenced by the time from onset of symptoms to diagnosis, the primary source of infection, and the number of abscesses [4, $6,7]$. Specific to a pediatric population, edema and midline shift have been identified as risk factors for worse prognosis [4]. One expert has suggested that cognitive decline is seen in $30-56 \%$ of all cerebral abscess patients [1]. The present case is the first to illustrate post-acute neuropsychological functioning in an adolescent patient with bilateral cerebral abscesses.

\section{Case Report}

The patient is a 16-year-old, right-handed male with a history of complex congenital heart disease (CHD). Born in Saudi Arabia, he underwent three surgeries in India related to his CHD and was raised speaking three languages at home-English, Hindi, and Malayalam — while attending an English-language school. Birth, developmental, and psychiatric history was unremarkable. At 11 years of age, he immigrated to the United States with his family. Six months prior to his neuropsychological evaluation, he presented to the emergency department with a 24-hour history of lowgrade fever, left-sided weakness, balance problems, fatigue, and disruptions in consciousness. CT and MRI studies of the brain showed left-to-right midline shift secondary to bilateral rim-enhancing intra-axial abscesses located in the left ventral thalamus and the right superior frontal gyrus, with surrounding white matter edema (Fig. 1). T2-weighted images indicated mass effect on the fornix and the left optic tract with edema extending into the posterior limb of the

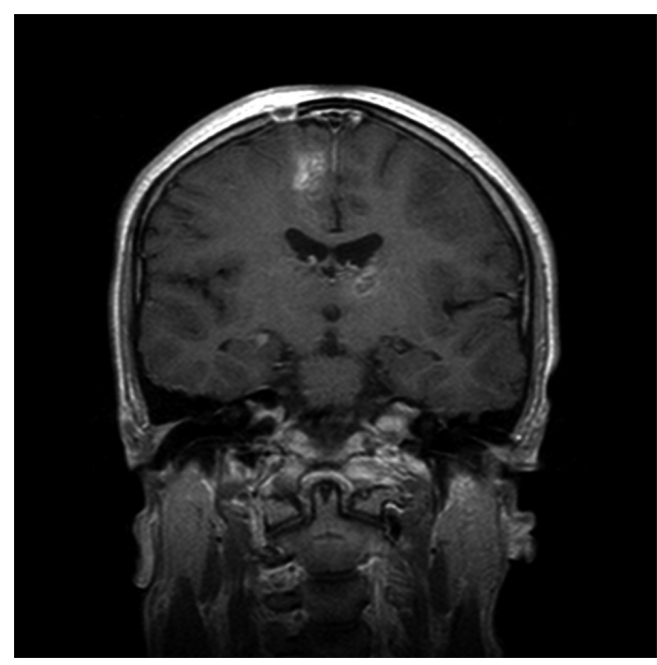

Figure 3. T1-weighted contrast-enhanced coronal image at 8 weeks post-operative shows minimal residual inflammatory enhancement around the contracted abscess cavities. Edema is nearly resolved in the left thalamus and is significantly diminished around the right frontal abscess.

internal capsule (Fig. 2). The surgical pathology report indicated the presence of Streptococcus intermedius in the thalamic abscess fluid. The abscesses were drained bilaterally via burr holes, and the patient was treated with antibiotics and a six-week course of steroids. A follow-up MRI eight weeks post-operative demonstrated marked contraction of the ring-enhancing abscess collections, near complete resolution of edema in the left thalamus, and significantly diminished edema around the small residual right frontal abscess cavity (Fig. 3). The follow-up MRI also showed enlargement of cerebral sulci and ventricles consistent with volume loss, as well as minimal gliosis of the cerebellar hemispheres.

The patient presented for neuropsychological evaluation six months following treatment of the abscesses due to difficulties with processing speed, memory, and sleep since his hospitalization. Additionally, his grades had dropped from historically straight-A's to primarily B's and a D in his Honors math class. He was in the eleventh grade at the time of the evaluation and was not receiving any formal accommodations. Upon presenting for evaluation, the patient appeared to be mildly depressed, demonstrating limited range of affect and minimal speech. He demonstrated satisfactory attention, task perseverance, and cooperation, thus supporting the validity of the neuropsychological testing results. As noted in Table 1, the testing results indicated below average overall cognitive ability, incongruent with his historical level of functioning. Within this context, performance was below average or impaired on measures of nonverbal reasoning, motor-based processing speed, visual-motor integration, bilateral fine motor speed, delayed visual and verbal memory, and select executive functions such as visual organization, cognitive flexibility, and planning/organization 
Table 1. Neuropsychological Test Results

\begin{tabular}{|c|c|}
\hline Measure & Standard Score \\
\hline \multicolumn{2}{|l|}{ Wechsler Intelligence Scale for Children IV } \\
\hline Full-Scale IQ & 79 \\
\hline Verbal Comprehension Index & 98 \\
\hline Perceptual Reasoning Index & 71 \\
\hline Working Memory Index & 91 \\
\hline Processing Speed Index & 73 \\
\hline \multicolumn{2}{|l|}{ Woodcock-Johnson III Tests of Achievement } \\
\hline Calculation & 102 \\
\hline Math Fluency & 94 \\
\hline Math Calculation Skills & 100 \\
\hline Letter-Word Identification & 103 \\
\hline Reading Fluency & 87 \\
\hline Spelling & 119 \\
\hline Handwriting & 125 \\
\hline Academic Skills & 110 \\
\hline Symbol-Digit Modalities Test & 98 \\
\hline Expressive Vocabulary Test, Second Edition & 95 \\
\hline \multicolumn{2}{|c|}{ California Verbal Learning Test, Children's Version } \\
\hline List A Total Trials 1 - 5 & 85 \\
\hline List A Trial 1 Free Recall & 85 \\
\hline List A Trial 5 Free Recall & 85 \\
\hline List B Free Recall & 78 \\
\hline List A Short-Delay Free Recall & 85 \\
\hline List A Short-Delay Cued Recall & 85 \\
\hline List A Long-Delay Free Recall & 78 \\
\hline List A Long-Delay Cued Recall & 85 \\
\hline Correct Recognition Hits & 85 \\
\hline Discriminability & 93 \\
\hline Perseverations & 85 \\
\hline Intrusions & 93 \\
\hline \multicolumn{2}{|l|}{ Children's Memory Scale } \\
\hline Stories - Immediate & 75 \\
\hline Stories - Delayed & 75 \\
\hline Stories - Delayed Recognition & 90 \\
\hline \multicolumn{2}{|l|}{ NEPSY-II Memory for Designs } \\
\hline Immediate & 85 \\
\hline Delayed & 80 \\
\hline \multicolumn{2}{|l|}{ Rey-Osterrieth Complex Figure Test } \\
\hline Copy & 50 \\
\hline Delay & 71 \\
\hline Hooper Visual Organization Test & 95 \\
\hline \multicolumn{2}{|l|}{ Purdue Pegboard } \\
\hline Right Hand & 55 \\
\hline Left Hand & 67 \\
\hline Both Hands & 85 \\
\hline \multicolumn{2}{|l|}{ DKEFS Trail Making } \\
\hline Visual Scanning & 90 \\
\hline Number Sequencing & 85 \\
\hline Letter Sequencing & 75 \\
\hline Number-Letter Sequencing & 55 \\
\hline Motor Speed & 80 \\
\hline \multicolumn{2}{|l|}{ DKEFS Verbal Fluency } \\
\hline Letter Fluency & 85 \\
\hline Category Fluency & 70 \\
\hline Category Switching Correct & 80 \\
\hline Category Switching Accuracy & 90 \\
\hline \multicolumn{2}{|l|}{ Conners' Continuous Performance Test II } \\
\hline \# Omissions & 98 \\
\hline \# Commissions & 110 \\
\hline Hit Rate & 95 \\
\hline Hit Rate Standard Error & 98 \\
\hline Variability & 91 \\
\hline Detectability & 112 \\
\hline Response Style & 94 \\
\hline Perseverations & 94 \\
\hline Hit Rate Block Change & 98 \\
\hline Hit Rate Standard Error Block Change & 91 \\
\hline Hit Rate ISI Change & 103 \\
\hline Hit Rate Standard Error ISI Change & 107 \\
\hline
\end{tabular}

Note. DKEFS: Delis-Kaplan Executive Function System. 
Table 2. Behavior Rating Inventory of Executive Functioning (BRIEF)

\begin{tabular}{lcc}
\hline BRIEF Subscale & Parent T-Score & Teacher T-Score \\
\hline Inhibit & 60 & 48 \\
Shift & 61 & 50 \\
Emotional Control & 60 & 45 \\
Behavioral Regulation Index & 62 & 47 \\
Initiate & 59 & $88^{*}$ \\
Working Memory & $66^{*}$ & $70^{*}$ \\
Plan/Organize & 59 & $78^{*}$ \\
Organization of Materials & 43 & 51 \\
Monitor & 57 & $73^{*}$ \\
Metacognition Index & 58 & $77^{*}$ \\
Global Executive Composite & 60 & $67^{*}$ \\
\hline
\end{tabular}

Note. An asterisk ( $\left.{ }^{*}\right)$ indicates a clinically significant score.

(Tables 1, 2). By contrast, motor-free processing speed, language, visual-spatial recognition, and sustained attention were intact. Additionally, basic academic skills were at or above age expectations. The patient was described as being at-risk for anxiety and depression (Table 3) per his teacher's responses on a questionnaire. Those ratings were supported by the aforementioned observations of his affect during the evaluation.

\section{Discussion}

CHD has been noted in association with cerebral abscess in $37-50 \%$ of pediatric populations $[4,6]$. It is also notable that in a study of children diagnosed with cerebral abscess, mean age at diagnosis was significantly lower for children with history of CHD, with the male:female ratio being nearly equal [6]. Palliative shunt surgery for CHD did not reduce the risk of cerebral abscess [6]. The mechanism by which CHD represents a risk factor for cerebral abscess is uncertain, but potential explanations include polycythemia, altered permeability of the blood-brain barrier, and right-toleft cardiovascular shunts which prevent normal physiologic bacterial phagocytosis in the lungs [4]. These and other potential complications associated with CHD (e.g., hypoxia) represent potential intrinsic risk factors for neuropsychological functioning. Thus, the importance of obtaining a thorough history detailing premorbid functioning cannot be underestimated when conducting neuropsychological evalu- ations of patients with a history of CHD. In the present case, both clinical interviews and report cards clarified that the patient was functioning at a high level prior to the development of the bilateral cerebral abscesses.

Based on reports of the patient's premorbid functioning, his neuropsychological profile at the time of the evaluation suggested significant compromise, likely reflecting the lingering impact of the bilateral cerebral abscesses six-months status post. In addition to those abscesses, the patient's history of complex congenital heart disease and consequent cyanosis at birth represent risk factors for impairments in brain development and cognitive functioning. Depressed mood, which was reported by his father and teacher and was observed during the evaluation, may exacerbate neuropsychological difficulties.

Due to the rarity of cerebral abscess, there is no known typical neuropsychological profile of patients in either acute or recovery stages associated with this condition. The literature is limited to a single study of 20 patients in the adult population [2]. That study found that $65 \%$ of their sample demonstrated long-term cognitive deficits, predominantly in verbal memory and executive functioning. Additionally, $65 \%$ of the sample demonstrated signs of irritability, depression, and/or aggression. Overall, the neuropsychological profile was characterized as a generalized subcortical pattern rather than a focal profile; this generalized profile was theorized to be the result of widespread inflammatory processes within the cerebrum [2].

Despite those findings, the present case suggests that the 
Table 3. Behavior Assessment System for Children, Second Edition (BASC-2)

\begin{tabular}{|c|c|c|c|}
\hline BASC-2 Subscale & Parent T-Score & Teacher T-Score & Self T-Score \\
\hline \multicolumn{4}{|l|}{ Clinical Scales } \\
\hline Hyperactivity & 54 & 41 & 33 \\
\hline Aggression & 40 & 43 & -- \\
\hline Conduct Problems & 44 & 42 & -- \\
\hline Anxiety & 50 & $64 *$ & 35 \\
\hline Depression & 49 & $60 *$ & 40 \\
\hline Somatization & 55 & 44 & -- \\
\hline Atypicality & $76^{* *}$ & 54 & 42 \\
\hline \multicolumn{4}{|l|}{ Clinical Scales } \\
\hline Withdrawal & 52 & $86^{* *}$ & -- \\
\hline Attention Problems & 53 & 54 & 36 \\
\hline Learning Problems & -- & $67^{*}$ & -- \\
\hline Attitude to School & -- & -- & 35 \\
\hline Attitude to Teachers & -- & -- & 39 \\
\hline Locus of Control & -- & -- & 37 \\
\hline Social Stress & -- & -- & 34 \\
\hline Sense of Inadequacy & -- & -- & 44 \\
\hline \multicolumn{4}{|l|}{ Adaptive Scales } \\
\hline Relations with Parents & -- & -- & 62 \\
\hline Interpersonal Relations & -- & -- & 59 \\
\hline Self-Esteem & -- & -- & 57 \\
\hline Self-Reliance & -- & -- & $33^{*}$ \\
\hline Adaptability & 49 & 42 & -- \\
\hline Social Skills & $33^{*}$ & $30 * *$ & -- \\
\hline Leadership & $40^{*}$ & $33 *$ & -- \\
\hline Activities of Daily Living & $40^{*}$ & -- & -- \\
\hline Study Skills & -- & 40 & -- \\
\hline Functional Communication & $39^{*}$ & $35^{*}$ & -- \\
\hline \multicolumn{4}{|l|}{ Composite Indices } \\
\hline School Problems & -- & $61^{*}$ & 30 \\
\hline Externalizing Problems & 46 & 41 & -- \\
\hline Internalizing Problems & 52 & 57 & 35 \\
\hline Inattention/Hyperactivity & -- & -- & 32 \\
\hline Behavioral Symptoms Index & 55 & 58 & -- \\
\hline Emotional Symptoms Index & -- & -- & 42 \\
\hline Adaptive Skills & $38^{*}$ & $34 *$ & -- \\
\hline Personal Adjustment & -- & -- & 54 \\
\hline
\end{tabular}

Note. A single asterisk (*) indicates an at-risk score. Two asterisks $\left(^{(*}\right)$ indicate a clinically significant score. 
neuropsychological consequences of cerebral abscess still may largely depend on the area of the brain that is affected. In the present case, abscesses in the left ventral thalamus may have contributed to difficulties with fine motor speed and visual-motor integration, in part due to lingering neuroanatomical consequences of mass effect on the left optic tract. Furthermore, research suggests that the left ventral thalamus plays a role in verbal memory and language production [8], which could explain some of this patient's difficulties with memory and limited speech. Additionally, this patient's deficits in executive functions may have been impacted by his right frontal abscess. Furthermore, and more in line with the findings of the prior study, subcortical white matter injury resulting from mass effect may have played a role in this patient's motor-based processing speed deficits. Finally, right hemisphere injury resulting from left-to-right midline shift of the brain may partially account for this patient's deficits in nonverbal reasoning and visual memory, the latter particularly due to injury to the fornix [9].

In neuropsychological evaluation of cerebral abscess patients, test selection based largely on the referral question is of the utmost importance. Additionally, test selection should be guided by the localization of the abscess. Even six months post-operatively, the present case demonstrated a significant number of deficits related to known functions of the regions in which the abscesses were located. However, test selection based on abscess localization should not be to the exclusion of other domains. For example, the present case demonstrated significant memory deficits despite minimal injury to the hippocampus. Additionally, based on the findings of both this case study and the prior study, an investigation of fine motor functioning may be warranted even if it is not a referral question, as subcortical white matter injury resulting from mass effect is likely to be a common mechanism of neuroanatomical damage from cerebral abscess.

Given that this is the first neuropsychological study of cerebral abscess published in North America and only the second to be published inclusive of other countries, additional research is greatly needed, moving beyond case studies to analyses of neuropsychological findings in larger samples of both adult and pediatric populations. The fact that this patient's deficits lingered six months post-acutely may indicate some degree of permanent compromise, although longitudinal neuropsychological follow-up would be needed to confirm this. However, the possibility of permanent compromise raises an important question: what is the typical neuropsychological outcome-both acute and long-term - of cerebral abscess? For example, fine motor functioning may be impaired regardless of the location of the abscess. Clearly, more research is needed to better understand both acute and post-acute neuropsychological outcomes of cerebral abscess, particularly in the rare case of bilateral cerebral abscesses.

\section{Disclosures}

We have no conflicts of interest to report.

\section{Disclaimers/Financial Support}

None.

\section{References}

1. Bernardini GL. Diagnosis and management of brain abscess and subdural empyema. Curr Neurol Neurosci Rep. 2004;4(6):448-456.

2. Visani P, Schmutzhard E, Trinka E, Pfausler B, Benke T. Subcortical deficit pattern after brain abscess: a neuropsychological study. Eur J Neurol. 2006;13(6):599-603.

3. Mathisen GE, Johnson JP. Brain abscess. Clin Infect Dis. 1997;25(4):763-779; quiz 780-761.

4. Atiq M, Ahmed US, Allana SS, Chishti KN. Brain abscess in children. Indian J Pediatr. 2006;73(5):401-404.

5. Rosenblum ML, Hoff JT, Norman D, Weinstein PR, Pitts L. Decreased mortality from brain abscesses since advent of computerized tomography. J Neurosurg. 1978;49(5):658-668.

6. Aebi C, Kaufmann F, Schaad UB. Brain abscess in childhood--long-term experiences. Eur J Pediatr. 1991;150(4):282-286.

7. Seydoux C, Francioli P. Bacterial brain abscesses: factors influencing mortality and sequelae. Clin Infect Dis. 1992;15(3):394-401.

8. Johnson MD, Ojemann GA. The role of the human thalamus in language and memory: evidence from electrophysiological studies. Brain Cogn. 2000;42(2):218-230.

9. Tsivilis D, Vann SD, Denby C, Roberts N, Mayes AR, Montaldi D, Aggleton JP. A disproportionate role for the fornix and mammillary bodies in recall versus recognition memory. Nat Neurosci. 2008;11(7):834-842. 